\title{
Lifestyle Patterns and Weight Status in Spanish Adults: The ANIBES Study
}

\author{
Carmen Pérez-Rodrigo ${ }^{1}$, Marta Gianzo-Citores ${ }^{2}$, Ángel Gil ${ }^{3,4}$, Marcela González-Gross ${ }^{4,5}$, \\ Rosa M. Ortega ${ }^{6}$, Lluis Serra-Majem ${ }^{4,7}$, Gregorio Varela-Moreiras ${ }^{8,9}$ and \\ Javier Aranceta-Bartrina 4,10,*
}

1 FIDEC Foundation, University of the Basque Country (UPV/EHU), Gurtubay s/n, 48010 Bilbao, Spain; carmenperezrodrigo@gmail.com

2 Department of Physiology, Faculty of Medicine and Nursery, University of the Basque Country (UPV/EHU), Bo Sarriena s/n, Leioa, 48940 Bizkaia, Spain; martagnz_2@hotmail.com

3 Department of Biochemistry and Molecular Biology II, Institute of Nutrition and Food Sciences, Centre of Biomedical Research, University of Granada, Campus de la Salud, Avda. del Conocimiento, Armilla, 18100 Granada, Spain; agil@ugr.es

4 CIBEROBN, Biomedical Research Networking Center for Physiopathology of Obesity and Nutrition, Carlos III Health Institute, 28029 Madrid, Spain; marcela.gonzalez.gross@upm.es (M.G.-G.);

lluis.serra@ulpgc.es (L.S.-M.)

5 ImFINE Research Group, Department of Health and Human Performance, Universidad Politécnica de Madrid, C/Martín Fierro 7, 28040 Madrid, Spain

6 Department of Nutrition, Faculty of Pharmacy, Complutense University of Madrid, Plaza Ramón y Cajal s/n, 28040 Madrid, Spain; rortega@ucm.es

7 Research Institute of Biomedical and Health Sciences, Faculty of Health Sciences, University of Las Palmas de Gran Canaria, Paseo Blas Cabrera Felipe "Físico" s/n, 35016 Las Palmas de Gran Canaria, Spain

8 Department of Pharmaceutical and Health Sciences, Faculty of Pharmacy, CEU San Pablo University, Urb. Montepríncipe, Crta. Boadilla Km. 5.3, Boadilla del Monte, 28668 Madrid, Spain; gvarela@ceu.es or gvarela@fen.org.es

9 Spanish Nutrition Foundation (FEN), C/General Álvarez de Castro 20. 1a pta, 28010 Madrid, Spain

10 Department of Food Sciences and Physiology, University of Navarra, C/Irunlarrea 1, 31008 Pamplona, Spain

* Correspondence: jaranceta@unav.es or javieraranceta@bizkaia.eu; Tel.: +34-608-580924

Received: 10 April 2017; Accepted: 8 June 2017; Published: 14 June 2017

Abstract: Limited knowledge is available on lifestyle patterns in Spanish adults. We investigated
dietary patterns and possible meaningful clustering of physical activity, sedentary behavior, sleep
time, and smoking in Spanish adults aged 18-64 years and their association with obesity. Analysis
was based on a subsample ( $n=1617$ ) of the cross-sectional ANIBES study in Spain. We performed
exploratory factor analysis and subsequent cluster analysis of dietary patterns, physical activity,
sedentary behaviors, sleep time, and smoking. Logistic regression analysis was used to explore the
association between the cluster solutions and obesity. Factor analysis identified four dietary patterns,
"Traditional DP", "Mediterranean DP", "Snack DP" and "Dairy-sweet DP". Dietary patterns, physical
activity behaviors, sedentary behaviors, sleep time, and smoking in Spanish adults aggregated
into three different clusters of lifestyle patterns: "Mixed diet-physically active-low sedentary lifestyle
pattern", "Not poor diet-low physical activity-low sedentary lifestyle pattern" and "Poor diet-low physical
activity-sedentary lifestyle pattern". A higher proportion of people aged 18-30 years was classified into
the "Poor diet-low physical activity-sedentary lifestyle pattern". The prevalence odds ratio for obesity in
men in the "Mixed diet-physically active-low sedentary lifestyle pattern" was significantly lower compared
to those in the "Poor diet-low physical activity-sedentary lifestyle pattern". Those behavior patterns
are helpful to identify specific issues in population subgroups and inform intervention strategies.
The findings in this study underline the importance of designing and implementing interventions
that address multiple health risk practices, considering lifestyle patterns and associated determinants. 
Keywords: cluster analysis; dietary patterns; lifestyle patterns; physical activity; sedentary behavior; obesity; adults

\section{Introduction}

Overweight and obesity have progressively increased during the last decades and have become a major issue in public health, both in developed and developing economies across the five continents [1,2]. In Spain, recent data report that more than half of adults aged 18-64 years are classified as overweight or obese $[3,4]$. This is particularly worrying for the negative impact of this condition on health and quality of life $[2,5,6]$. In fact, the Global Burden of Disease project highlights that high body mass index (BMI) values were among the main risk factors driving most death and disability combined in the country in 2015 [7].

Overweight and obesity result from an imbalance between energy intake and expenditure. The role of diet in obesity is complex. Most research in this area has focused on specific foods and nutrients [8,9]. Nevertheless, the analysis of food patterns is particularly interesting, since foods are usually consumed in combinations and those may have synergistic, antagonistic, or moderating effects [10].

In addition, to date, it is acknowledged that joint interactions of multiple variables acting at different levels influence weight gain, such as lifestyles [11-13], sleep, including rhythm, duration or quality of sleep [14,15], eating behaviors [16], socioeconomic level, education, and other factors $[4,11]$. Data-driven methods explore the similarities between different food options in specific population groups [8]. Specifically, cluster analyses include several techniques aimed at grouping together individuals sharing a number of features or similar lifestyles. This classification allows for a better understanding of the influences of behaviors and lifestyles, as well as the potential cumulative effects of an unhealthy combination of those factors on the development of overweight and obesity [16]. Research of dietary patterns and the potential combination of those with other lifestyles can contribute to identifying effective strategies for the prevention of overweight and obesity among adults, as well as its social and health consequences [8].

Considering the above, the objectives of this paper are (a) to identify food patterns in the Spanish adult population; (b) to investigate if energy balance-related behaviors tend to assemble into meaningful patterns in Spanish adults; (c) to describe existing relationships between socio-demographical factors and different lifestyle patterns; and, finally; (d) to analyze the potential association of those correlates with excess body weight.

\section{Materials and Methods}

The data for this analysis were obtained from the ANIBES study, an observational cross-sectional study conducted on a random sample of the Spanish population aged 9-75 years. The aims and procedures used in ANIBES have been previously reported [17,18].

Briefly, the sample design of the ANIBES study was based on the Spanish population census 2012 according to sex, age, residence, and regional population size. A stratified multistep sampling procedure was used, with random selection of addresses in the municipalities and age and sex quotas for individuals within households. Interlocked quotas were established for age in the regions and size of habitat within the region. The sample selection procedure was based on random paths; 128 sampling points were selected.

The final sample of the study consisted of 2009 individuals (1013 men, 50.4\%, 996 women, $49.6 \%$ ). In addition, a boost sample was recruited for the younger groups (9-12 years; $13-17$ years, and 18-24 years) in order to ensure at least 200 individuals in each age group. Data for individuals aged 18-64 years were used for this analysis. That age range is meaningful from a risk prevention point of view, since it includes adults in the active working life span, thus, relevant when designing targeted preventive and health promotion strategies, considering different settings, such as workplaces, 
primary health care, and community-based interventions. In addition, the focus on the 18-64 year-old range was also relevant for comparison with previous studies conducted in Spain [19]. Data were collected between mid-September and mid-November 2013.

The final protocol of the study was approved by the ethical research committee of the Community of Madrid, Spain.

\subsection{Measurements}

\subsubsection{Lifestyle Factors}

Diet

Dietary intake was assessed by means of a face-to-face 24-h diet recall interview assisted by a food picture atlas to estimate portion sizes. In addition participants completed a three-day food record aided by a tablet device (Samsung Galaxy Tab 2 7.0, Samsung Electronics, Suwon, South Korea), two consecutive weekdays and one weekend day, recording all foods and beverages consumed at home and away from home. Processing of food record inputs, coding and data cleaning has been extensively reported elsewhere $[17,18]$. Energy and nutrient intakes were calculated using new specifically-developed software, (VD-FEN 2.1 software -Dietary Evaluation Programme, Spanish Nutrition Foundation, Madrid, Spain) for the ANIBES study [20]. Consumption of all food and beverages was arranged into 16 food groups, 45 subgroups, and 754 food items, for in-depth analysis, based on the structure of the food composition database considering similarities in nutrient profile (Supplementary Materials Table S1).

Physical Activity

Physical activity data were collected by face-to-face interview using the validated International Physical Activity Questionnaire (IPAQ) [21]. Total minutes per week were computed for moderate to vigorous physical activity based on the IPAQ guidelines for data processing and analyses [22]. Data were cleaned and truncated based on IPAQ guidelines and previous research [23]. Additionally, the total minutes per week of commuting-related physical activity (walking, biking) were computed. IPAQ data was used for this analysis. Z-scores of minutes per week for each type of activity were calculated.

\section{Sleep Duration}

Sleep habits included the number of hours slept per night, on average, as reported by each individual.

Smoking

Smoking habits were assessed by different items in the protocol, including "During the past year, how many cigarettes per day did you smoke on average?"

\subsubsection{Body Measurements}

Anthropometric measurements were taken individually by trained interviewers, following international standard procedures previously tested in two pilot studies [24], reported in detail elsewhere $[17,18]$. Body mass index (BMI) was calculated as body weight in kilograms divided by the square of body height in meters. Overweight status was defined as BMI $\geq 25$; obesity as BMI $\geq 30$.

\subsubsection{Covariates}

Education

The education levels were established in accordance with the Spanish educational system. 
After preliminary analysis of the distribution of the variable, categories were collapsed and recoded into a three-point scale, as follows: (1) low (less than seven years of education; primary school or less); (2) medium (7-12 years of education; lower to higher secondary education); and (3) high (13 years or more of education; higher vocational, college, and university studies).

\section{Geographical Area}

Geographical area in the country was collapsed into four different categories: north-northwest region; eastern region; central region and southern region.

\subsection{Data Cleaning}

Detailed data cleaning procedures have been previously described $[17,18]$. Participants were considered fully eligible after a verified quality check of the input from the tablet device of adequately completed three-day food records. After data cleaning stages, individuals remained in the database if they had successfully completed both face-to-face interviews during fieldwork and had measured weight, height, and waist circumference data. Of the initial sample of 1655 recruited individuals aged 18-64 years, 1617 individuals satisfied the inclusion criteria and had complete data for all of the variables included in this analysis.

\subsection{Data Analysis}

All statistical tests were performed using IBM SPSS Statistics for Windows, Version 22.0 (IBM Corp., Armonk, NY, USA). Descriptive statistics were computed for each variable.

\subsubsection{Dietary Patterns}

Exploratory factor analysis was performed to identify underlying dietary patterns, using the average food weight ( $\mathrm{g} /$ day) consumed by each individual (three-day food record plus one-day 24-h recall) from 38 food groups as input variables. Food groups were used to further collapse dietary intake data in order to avoid missing data from non-consumers of episodically consumed foods. Z-scores for each food group were calculated to prevent the components being dominated by the foods that provide the highest amounts. Bartlett's test of sphericity and the Kaiser-Meyer-Olkin (KMO) measure of sampling adequacy were used to verify the appropriateness of factor analysis. Factors were also orthogonally rotated (the varimax option) to enhance the difference between loadings, which allowed for easier interpretability.

Factors were retained based on the following criteria: factor eigenvalue $>1.20$, identification of a break point in the scree plot, the proportion of variance explained, and factor interpretability [25].

The strength and direction of the associations between patterns and food groups were described through a rotated factor loading matrix. Food groups with factor loadings $>0.30$ and communality $>0.20$ were retained in the patterns identified. The factor score for each pattern was constructed by summing the observed intakes of the component food items weighted by the factor loading. A high factor score for a given pattern indicated high intake of the foods constituting that food factor, and a low score indicated low intake of those foods.

\subsubsection{Lifestyle Patterns}

To identify clusters with similar dietary patterns, physical activities, sedentary activities, sleeping habits and smoking a combination of hierarchical and non-hierarchical clustering analysis was used [26]. The variables used had different arithmetic scales; thus, Z-scores were calculated to standardize the dataset before clustering, to avoid a greater contribution to the distance of variables having larger ranges than variables with smaller ranges. Univariate and multivariate outliers (>3SD) were removed. First, hierarchical cluster analysis was performed using Ward's method, based on squared Euclidian distances. Several possible cluster solutions were identified and compared to inform 
the next step, considering the coefficients and fusion level. A non-hierarchical k-means clustering procedure was used, specifying the number of clusters identified in the first step, using a random initial seed and 10 iterations in order to further refine the preliminary solution by optimizing the classification. The final cluster solution was selected based on interpretability and the percent of the study population in each cluster. Reliability and stability of the final cluster solution was tested by randomly taking a subsample (50\%) of the total sample and repeating the analyses on this subsample. To check agreement, a kappa statistic was calculated between the cluster solutions of the subsample and that of the total sample.

Pearson's chi-square tests were used to investigate the differences in cluster distribution by gender, age group, education level, geographical area, and BMI status. One-way ANOVA was used to compare physical activities, sedentary behaviors and sleep time across clusters stratified by gender and age group. General linear models were used to estimate multivariate means for food consumption and dietary pattern scores across clusters adjusted for age and energy intake. Binary logistic regression analysis was used to explore the prevalence odds ratios for obesity and overweight among lifestyle patterns. The models were adjusted for energy intake, sex, age, educational level, and geographical area. Statistical tests were two-tailed with a $5 \%$ level of significance.

\section{Results}

\subsection{Sample Characteristics}

After exclusion of outliers and participants with incomplete data, 1617 subjects aged 18-64 years, 781 men and 836 women, were included in the analyses (Table S2). Some $38 \%$ of the sample was classified in the BMI range 25-29.9 and $21.6 \%$ had BMI values $\geq 30$. There was no significant difference between men and women in age distribution, level of education, or geographical area. However, overweight and obesity rates were significantly higher in men than women.

\subsection{Dietary Patterns}

Dietary patterns were computed for the entire sample. Bartlett's test of sphericity and $\mathrm{KMO}=0.591$ supported the appropriateness of factor analysis. Four major factors were extracted, which explained $33.1 \%$ of the variance in the model. The first dietary pattern (DP) was labeled "Traditional DP" which had the highest loading on olive oil and vegetables, high scores on fish, meat, and fruit, and negative scores on pasta and so-called "pre-cooked" foods, which include food items such as croquettes and other processed foods usually prepared deep-fried for consumption. A DP labeled "Mediterranean DP" had high scores on water, fruit, yoghourt, fish, vegetables, cheese, and olive oil, and negative scores on meat and sugar sweetened beverages. A DP labeled "Snack DP" had high scores on bread, processed and cold meats, alcoholic beverages, salted snacks, cheese, and juices. Finally, "Dairy-sweet DP" had high scores on milk, sugar and sweets, cakes, pastry, and juices, and negative scores on alcoholic beverages. Overall, these patterns explained 33.07\% of the variance (Figure 1).

Mean factor scores for "Traditional DP" and "Mediterranean DP" were significantly higher in the oldest age group (50-64 years), while "Dairy-sweet DP" factor scores were significantly higher in the younger ones (18-30 years). Men had significantly higher scores than women for "Snack DP" adjusted for age and energy intake; "Mediterranean $D P$ " and "Dairy-sweet $D P$ " had significantly higher scores among women. "Mediterranean $D P^{\prime}$ " and "Traditional $D P$ " factor scores were significantly higher in people with a higher educational level.

Factor scores for the "Traditional DP" and "Dairy-sweet DP", adjusted for energy intake, age, and gender, were significantly higher in the north-northwest region, while "Mediterranean $D P$ " and "Snack DP" factor scores were significantly higher in the Eastern Mediterranean region. 


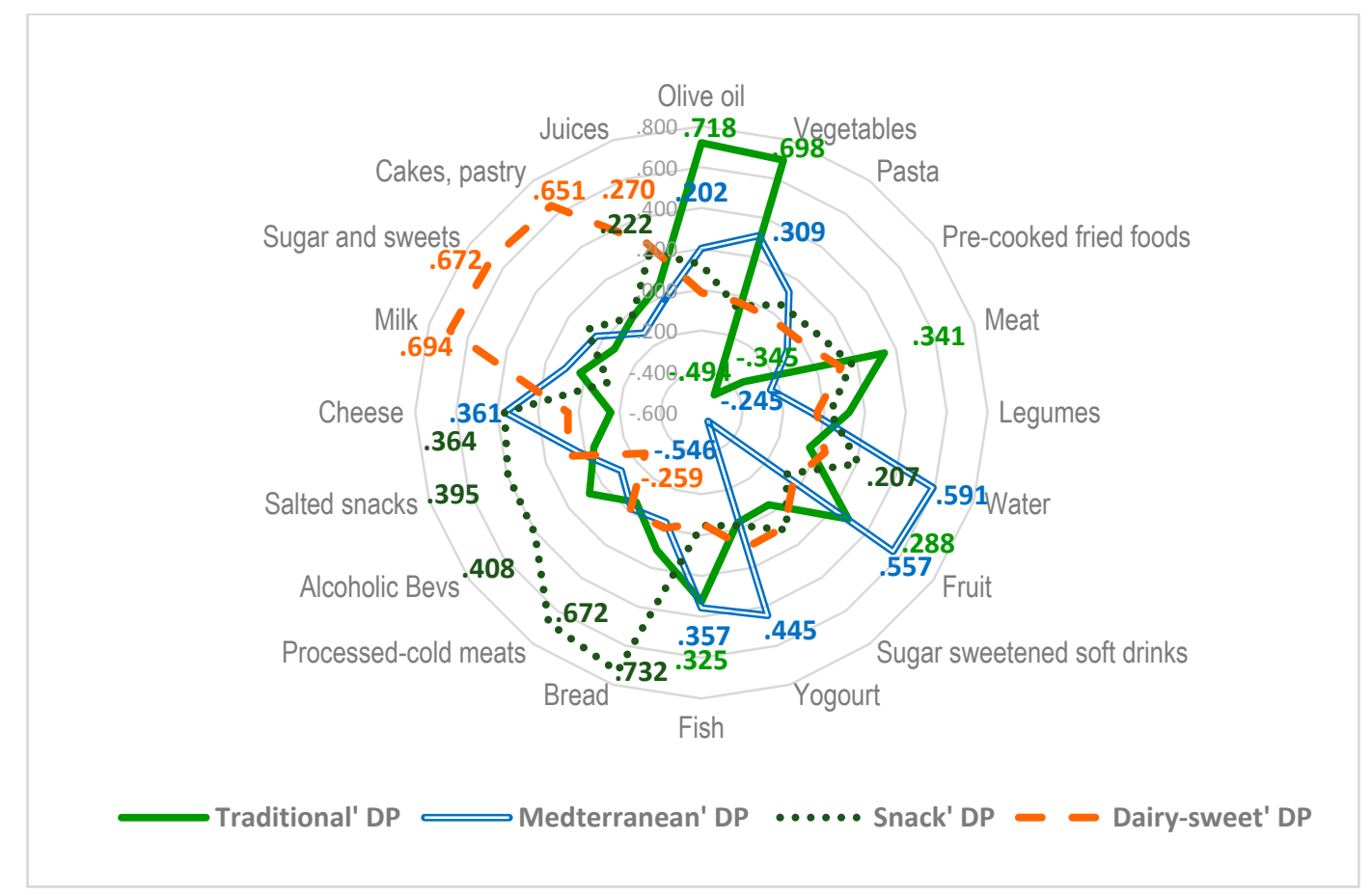

Figure 1. Factor loadings after varimax rotation on identified dietary patterns of food groups retained. Eigenvalues: Traditional DP $=2.15$; Mediterranean $D P=1.64$; Snack DP $=1.55 ;$ Dairy-sweet DP $=1.28$. Percent of variance explained: Traditional DP: 8.74\%; Mediterranean DP: 8.39\%; Snack DP: 8.19\%; Dairy sweet DP: $7.74 \%$. Total variance explained $33.07 \%$. Absolute values less than 0.20 are not shown.

\subsection{Lifestyle Patterns}

Based on the four identified DPs, minutes per week of vigorous, moderate physical activity, walking, biking, sedentary time, sleep duration on weekdays, and smoking habits, the three-cluster solution was found to be adequate and meaningful regarding the different patterns. The kappa statistic $(\kappa=0.94)$ suggested good agreement.

Differential characteristics of each cluster are identified by high (above 0 ) or low Z-scores (below 0) comparing cluster centers in Z-scores (Figure S1). Cluster 1, labeled "Mixed diet-physically active-low sedentary lifestyle pattern", drew a lifestyle pattern with high scores on the "Snack DP" $(Z$-score $=0.42)$, the "Mediterranean DP" $(Z$-score $=0.27)$, walking $(Z$-score $=0.58)$, vigorous physical activity $(Z$-score $=2.09)$ and moderate physical activity $(Z$-score $=0.33)$, combined with low scores on sedentary time $(Z$-score $=-0.40)$ and sleeping $(Z$-score $=-0.25)$. The second cluster, labeled "Not poor diet-low physical activity-low sedentary lifestyle pattern", had low scores on "Dairy-sweet DP" $(Z$-score $=-0.40)$, "Snack $D P$ " $(Z$-score $=-0.16)$, vigorous physical activity $(Z$-score $=-0.34)$ and sedentary time (Z-score $=-0.19)$. Finally, a third cluster labeled "Poor diet-low physical activity-sedentary lifestyle pattern" had high scores on "Dairy-sweet DP" (Z-score =1.09), "Snack DP" $(Z$-score = 0.19) and sedentary time $(Z$-score $=0.73)$ and scored negatively on moderate $(Z$-score $=-0.42)$ and vigorous physical activity $(Z$-score $=-0.26)$, as well as smoking $(Z$-score $=-0.21)$.

Characteristics of the subjects classified in the lifestyle patterns identified are described in Table 1. The "Mixed diet-physically active-low sedentary lifestyle pattern" included 13\% of the sample and a significantly higher proportion of men. The "Not poor diet-low physical activity-low sedentary lifestyle pattern" included $63.3 \%$ of the sample, a significantly higher proportion of women. The "Poor diet-low physical activity-sedentary lifestyle pattern" included $23.6 \%$ of the sample. 
Table 1. Gender, age group, educational level, geographical area, and BMI status by lifestyle pattern.

\begin{tabular}{|c|c|c|c|c|c|c|c|c|c|}
\hline \multirow[t]{2}{*}{ Characteristics } & \multicolumn{2}{|c|}{$\begin{array}{l}\text { Mixed Diet-Physically } \\
\text { Active-Low Sedentary } \\
\text { Lifestyle Pattern }\end{array}$} & \multicolumn{2}{|c|}{$\begin{array}{c}\text { Not Poor Diet-Low } \\
\text { Physical Activity-Low } \\
\text { Sedentary Lifestyle Pattern }\end{array}$} & \multicolumn{2}{|c|}{$\begin{array}{l}\text { Poor Diet-Low Physical } \\
\text { Activity-Sedentary } \\
\text { Lifestyle Pattern }\end{array}$} & \multirow{2}{*}{$\begin{array}{l}\text { All } \\
n\end{array}$} & \multirow[t]{2}{*}{$x^{2}$} & \multirow[t]{2}{*}{$p$} \\
\hline & $n$ & $\%$ & $n$ & $\%$ & $n$ & $\%$ & & & \\
\hline All & 210 & 13.0 & 1020 & 63.3 & 381 & 23.6 & 1611 & & \\
\hline \multicolumn{10}{|l|}{ Gender } \\
\hline Men & 151 & 71.9 & 423 & 41.5 & 204 & 53.5 & 778 & 70.1 & 0.000 \\
\hline Women & 59 & 28.1 & 597 & 58.5 & 177 & 46.5 & 833 & & \\
\hline \multicolumn{10}{|l|}{ Age group } \\
\hline $18-30$ years & 61 & 29.0 & 213 & 20.9 & 139 & 36.5 & 413 & 60.3 & 0.000 \\
\hline $31-49$ years & 110 & 52.4 & 487 & 47.7 & 183 & 48.0 & 780 & & \\
\hline 50-64 years & 39 & 18.6 & 320 & 31.4 & 59 & 15.5 & 418 & & \\
\hline Educational level & & & & & & & & 27.8 & 0.000 \\
\hline Primary or less & 55 & 26.2 & 304 & 29.8 & 73 & 19.2 & 432 & & \\
\hline Secondary & 95 & 45.2 & 507 & 49.7 & 189 & 49.6 & 791 & & \\
\hline Higher & 60 & 28.6 & 209 & 20.5 & 119 & 31.2 & 388 & & \\
\hline \multicolumn{10}{|l|}{ Geographical area } \\
\hline North-northwest & 36 & 17.1 & 166 & 16.3 & 74 & 19.4 & 276 & 4.7 & 0.577 \\
\hline Eastern-Mediterranean & 70 & 33.3 & 350 & 34.3 & 127 & 33.3 & 547 & & \\
\hline Center & 48 & 22.9 & 233 & 22.8 & 96 & 25.2 & 377 & & \\
\hline South & 56 & 26.7 & 271 & 26.6 & 84 & 22.0 & 411 & & \\
\hline \multicolumn{10}{|l|}{ BMI status } \\
\hline Normal weight & 88 & 41.9 & 385 & 37.7 & 177 & 46.5 & 650 & 17.3 & 0.002 \\
\hline Overweight & 90 & 42.9 & 386 & 37.8 & 136 & 35.7 & 612 & & \\
\hline Obese & 32 & 15.2 & 249 & 24.4 & 68 & 17.8 & 349 & & \\
\hline
\end{tabular}

Pearson's chi-square tests were used to investigate the differences in cluster distribution by gender, age group, education level, geographical area, and BMI status.

A higher percentage of people across age groups were classified into the "Not poor diet-low physical activity-low sedentary lifestyle pattern". However, a significantly lower proportion of individuals in the older age group (50-64 years) was classified in the "Mixed diet-physically active-low sedentary lifestyle pattern" and a higher proportion of people aged 18-30 years were classified into the "Poor diet-low physical activity-sedentary lifestyle pattern". The highest proportion of people with a lower educational level was classified into the "Not poor diet-low physical activity-low sedentary lifestyle pattern". There was no difference in the clusters by geographical area.

Prevalence rates of obesity were significantly higher among people allocated in the "Not poor diet-low physical activity-low sedentary lifestyle pattern" and overweight in the "Mixed diet-physically active-low sedentary lifestyle pattern", however, those differences were not significant in the stratified analysis by age and gender.

Table 2 describes physical activity behaviors, sedentary, sleep time on weekdays, smoking behavior, and dietary pattern Z-scores in the lifestyle patterns. Vigorous physical activity, moderate physical activity, walking time, as well as the Z-scores of the Mediterranean DP were significantly higher in men and women classified in the "Mixed diet-physically active-low sedentary lifestyle pattern". $Z$-scores for "Snack DP" were also higher in men in that lifestyle pattern. Men and women in the "Poor diet-low physical activity-sedentary lifestyle pattern" had significantly higher scores on the "Dairy-sweet $D P^{\prime \prime}$ and sedentary time.

Consumption of selected food groups and beverages by lifestyle pattern in men and women is described in Table 3. Consumption of fruit, pasta, olive oil, water and alcoholic beverages, particularly wine and beer, was significantly higher in men and women included in the "Mixed diet-physically active-low sedentary lifestyle pattern". Consumption of milk, cakes and pastry, sugar and sweets was significantly higher in men and women classified in the "Poor diet-low physical activity-sedentary lifestyle pattern". Men in this lifestyle pattern showed significantly higher consumption of pre-cooked deep fried foods and high alcoholic content beverages. Women in this pattern had significantly higher consumption of savory snacks, juices and sugar sweetened soft drinks beverages.

Prevalence of obesity was compared between lifestyle patterns, adjusting for gender, age, educational level, geographical area and energy intake (Table 4). The prevalence odds ratio (POR) for obesity in men, 0.52 (IC 95\% 0.29-0.92) allocated in the "Mixed diet-physically active-low sedentary 
lifestyle pattern" was significantly lower compared to those in the "Poor diet-low physical activity-sedentary lifestyle pattern".

Table 2. Physical activity behaviors, sedentary, sleep time on weekdays, smoking behavior, and dietary pattern scores in the lifestyle patterns by gender *

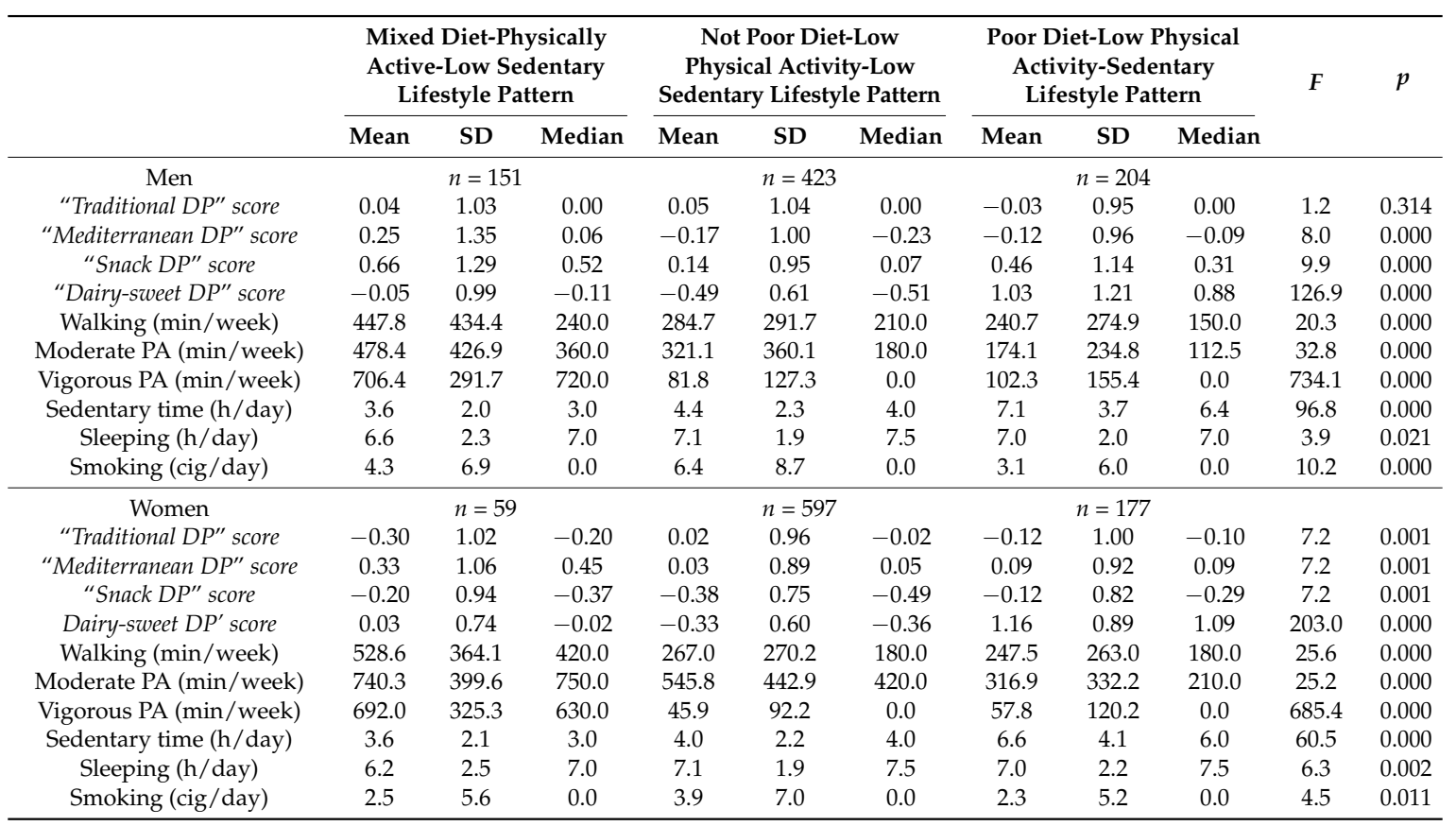

* Physical activities, sedentary time, sleep, and smoking compared by ANOVA considering age group. General linear models adjusted for age and energy intake to compare DP scores. 
Table 3. Consumption of selected food groups and beverages by lifestyle pattern in men and women.

\begin{tabular}{|c|c|c|c|c|c|c|c|c|c|c|c|}
\hline \multirow[b]{2}{*}{$\operatorname{Men}(n=781)$} & \multicolumn{3}{|c|}{$\begin{array}{l}\text { Mixed Diet-Physically } \\
\text { Active-Low Sedentary } \\
\text { Lifestyle Pattern }\end{array}$} & \multicolumn{3}{|c|}{$\begin{array}{c}\text { Not Poor Diet-Low Physical } \\
\text { Activity-Low Sedentary } \\
\text { Lifestyle Pattern }\end{array}$} & \multicolumn{3}{|c|}{$\begin{array}{c}\text { Poor Diet-Low Physical } \\
\text { Activity-Sedentary } \\
\text { Lifestyle Pattern }\end{array}$} & \multirow[t]{2}{*}{$F$} & \multirow[t]{2}{*}{$p$} \\
\hline & Mean & $\begin{array}{c}\text { SD } \\
(n=151)\end{array}$ & Median & Mean & $\begin{array}{c}\text { SD } \\
(n=423)\end{array}$ & Median & Mean & $\begin{array}{c}\text { SD } \\
(n=204)\end{array}$ & Median & & \\
\hline Vegetables (g/day) & 184.9 & 110.9 & 165.4 & 185.1 & 112.4 & 162.5 & 178.2 & 97.2 & 165.0 & 0.98 & 0.374 \\
\hline Fruit (g/day) & 183.1 & 231.0 & 136.7 & 145.0 & 172.7 & 97.5 & 139.5 & 144.0 & 103.9 & 3.24 & 0.040 \\
\hline Legumes (g/day) & 16.7 & 23.1 & 7.5 & 16.2 & 19.1 & 10.5 & 13.6 & 18.2 & 7.1 & 2.19 & 0.113 \\
\hline Meat (g/day) & 127.2 & 92.3 & 111.7 & 109.8 & 75.3 & 95.8 & 124.9 & 77.4 & 116.3 & 0.56 & 0.573 \\
\hline Processed and cold meats (g/day) & 55.8 & 46.6 & 44.3 & 42.5 & 36.0 & 34.2 & 50.3 & 39.1 & 45.9 & 2.46 & 0.086 \\
\hline Fish (g/day) & 73.6 & 90.8 & 47.7 & 62.5 & 66.9 & 39.3 & 55.8 & 57.1 & 35.4 & 3.01 & 0.050 \\
\hline Eggs (g/day) & 40.8 & 46.4 & 31.3 & 32.5 & 33.4 & 21.3 & 28.3 & 30.4 & 20.0 & 9.58 & 0.000 \\
\hline Milk (mL/day) & 155.5 & 122.9 & 139.7 & 125.6 & 100.7 & 115.0 & 267.5 & 178.5 & 249.4 & 60.42 & 0.000 \\
\hline Cheese (g/day) & 25.4 & 41.4 & 15.2 & 15.8 & 20.0 & 10.0 & 19.2 & 22.2 & 12.9 & 4.39 & 0.013 \\
\hline Yoghourt (g/day) & 62.3 & 74.5 & 41.7 & 42.3 & 64.2 & 0.0 & 46.2 & 62.0 & 20.8 & 3.62 & 0.027 \\
\hline Pasta (g/day) & 22.6 & 27.5 & 12.5 & 16.2 & 20.0 & 11.7 & 17.7 & 20.2 & 11.7 & 3.08 & 0.047 \\
\hline Bread (g/day) & 94.4 & 57.4 & 83.3 & 83.6 & 44.6 & 80.0 & 97.5 & 58.2 & 85.0 & 1.15 & 0.318 \\
\hline Cakes and pastry (g/day) & 30.3 & 36.1 & 16.7 & 21.1 & 25.7 & 11.7 & 57.8 & 46.3 & 50.2 & 44.80 & 0.000 \\
\hline Sugar and sweets (g/day) & 15.0 & 15.4 & 10.0 & 10.0 & 9.8 & 7.5 & 24.7 & 18.5 & 21.8 & 43.23 & 0.000 \\
\hline Pre-cooked foods (g/day) & 73.0 & 83.0 & 50.0 & 76.3 & 86.9 & 45.8 & 80.0 & 91.3 & 46.3 & 3.45 & 0.032 \\
\hline Savory snacks (g/day) & 6.1 & 12.1 & 0.0 & 4.7 & 10.1 & 0.0 & 7.5 & 14.3 & 0.0 & 0.51 & 0.603 \\
\hline Olive oil (mL/day) & 20.0 & 8.9 & 20.2 & 18.0 & 8.8 & 16.7 & 17.3 & 7.5 & 18.0 & 6.40 & 0.002 \\
\hline Juices (mL/day) & 71.0 & 123.9 & 0.0 & 40.0 & 79.8 & 0.0 & 88.6 & 175.4 & 0.0 & 1.51 & 0.221 \\
\hline Sugar sweetened soft drinks (mL/day) & 104.2 & 151.5 & 41.7 & 97.7 & 186.7 & 0.0 & 127.2 & 192.2 & 47.5 & 2.44 & 0.088 \\
\hline Water (mL/day) & 843.4 & 647.7 & 695.8 & 638.0 & 537.2 & 513.3 & 757.4 & 582.2 & 685.0 & 3.62 & 0.027 \\
\hline Alcoholic beverages (mL/day) & 186.1 & 259.4 & 71.7 & 176.4 & 241.2 & 58.3 & 102.7 & 181.9 & 0.0 & 14.33 & 0.000 \\
\hline Low alcohol content bevs (mL/day) & 1.6 & 5.7 & 0.0 & 2.8 & 11.6 & 0.0 & 3.4 & 19.4 & 0.0 & 2.95 & 0.05 \\
\hline High alcohol content bevs ( $\mathrm{mL} /$ day) & 184.5 & 257.6 & 71.7 & 173.6 & 238.8 & 55.8 & 99.3 & 177.3 & 0.0 & 14.31 & 0.00 \\
\hline
\end{tabular}


Table 3. Cont.

\begin{tabular}{|c|c|c|c|c|c|c|c|c|c|c|c|}
\hline \multirow[b]{2}{*}{ Women $(n=833)$} & \multicolumn{3}{|c|}{$\begin{array}{l}\text { Mixed Diet-Physically } \\
\text { Active-Low Sedentary } \\
\text { Lifestyle Pattern }\end{array}$} & \multicolumn{3}{|c|}{$\begin{array}{c}\text { Not Poor Diet-Low Physical } \\
\text { Activity-Low Sedentary } \\
\text { Lifestyle Pattern }\end{array}$} & \multicolumn{3}{|c|}{$\begin{array}{l}\text { Poor Diet-Low Physical } \\
\text { Activity-Sedentary } \\
\text { Lifestyle Pattern } \\
\end{array}$} & \multirow[t]{2}{*}{$F$} & \multirow[t]{2}{*}{$p$} \\
\hline & Mean & $\begin{array}{c}\text { SD } \\
n=59\end{array}$ & Median & Mean & $\begin{array}{c}\text { SD } \\
n=597\end{array}$ & Median & Mean & $\begin{array}{c}\text { SD } \\
n=177\end{array}$ & Median & & \\
\hline Vegetables (g/day) & 168.5 & 90.6 & 156.2 & 195.3 & 115.3 & 168.8 & 174.4 & 110.4 & 162.6 & 5.197 & 0.006 \\
\hline Fruit (g/day) & 186.5 & 175.6 & 152.5 & 162.4 & 173.0 & 111.5 & 143.8 & 147.0 & 110.0 & 0.929 & 0.395 \\
\hline Legumes (g/day) & 10.9 & 13.8 & 5.0 & 14.7 & 18.7 & 10.0 & 13.6 & 24.2 & 3.3 & 1.433 & 0.239 \\
\hline Meat (g/day) & 78.8 & 63.1 & 66.7 & 90.9 & 63.4 & 82.5 & 101.6 & 70.1 & 89.2 & 2.054 & 0.129 \\
\hline Processed and cold meats (g/day) & 37.1 & 31.5 & 29.7 & 33.5 & 30.1 & 26.7 & 37.5 & 32.2 & 29.2 & 1.582 & 0.206 \\
\hline Fish (g/day) & 55.4 & 50.4 & 45.0 & 59.8 & 62.9 & 40.0 & 58.1 & 65.9 & 38.3 & 0.142 & 0.868 \\
\hline Eggs (g/day) & 25.9 & 26.3 & 21.3 & 25.0 & 24.7 & 20.7 & 24.5 & 24.2 & 19.5 & 1.497 & 0.224 \\
\hline Milk (mL/day) & 175.0 & 104.7 & 175.0 & 148.2 & 103.1 & 141.7 & 271.2 & 138.3 & 255.0 & 72.001 & 0.000 \\
\hline Cheese (g/day) & 20.1 & 20.5 & 12.5 & 15.1 & 17.1 & 10.0 & 18.7 & 21.3 & 13.3 & 1.289 & 0.276 \\
\hline Yoghourt (g/day) & 53.9 & 56.7 & 30.8 & 43.7 & 57.2 & 20.8 & 51.4 & 58.7 & 22.5 & 2.550 & 0.079 \\
\hline Pasta (g/day) & 18.7 & 20.5 & 11.7 & 14.5 & 19.1 & 8.3 & 15.4 & 19.7 & 10.8 & 1.768 & 0.171 \\
\hline Bread (g/day) & 64.8 & 38.4 & 56.7 & 65.7 & 39.6 & 60.0 & 66.7 & 33.7 & 64.2 & 11.989 & 0.000 \\
\hline Cakes and pastry (g/day) & 31.6 & 37.1 & 20.7 & 20.9 & 22.6 & 15.0 & 56.3 & 40.9 & 50.0 & 54.345 & 0.000 \\
\hline Sugar and sweets (g/day) & 15.6 & 12.4 & 13.7 & 11.3 & 11.3 & 8.2 & 30.2 & 25.0 & 25.3 & 52.816 & 0.000 \\
\hline Pre-cooked foods (g/day) & 63.1 & 77.6 & 41.7 & 61.1 & 71.6 & 41.7 & 58.0 & 61.5 & 41.7 & 4.561 & 0.011 \\
\hline Savory snacks (g/day) & 5.9 & 9.6 & 0.0 & 3.4 & 7.4 & 0.0 & 9.0 & 14.8 & 2.0 & 7.045 & 0.001 \\
\hline Olive oil (mL/day) & 17.2 & 8.2 & 15.8 & 17.9 & 7.8 & 17.3 & 17.3 & 8.5 & 17.0 & 6.580 & 0.001 \\
\hline Juices (mL/day) & 51.0 & 80.5 & 0.0 & 32.5 & 60.0 & 0.0 & 61.3 & 86.8 & 13.3 & 3.348 & 0.036 \\
\hline Sugar sweetened soft drinks (mL/day) & 60.5 & 130.2 & 0.0 & 77.9 & 149.7 & 0.0 & 96.4 & 159.7 & 33.3 & 3.543 & 0.029 \\
\hline Water (mL/day) & 764.2 & 563.1 & 658.3 & 649.0 & 474.2 & 550.0 & 753.6 & 560.2 & 641.7 & 4.689 & 0.009 \\
\hline Alcoholic beverages (mL/day) & 84.4 & 132.5 & 30.0 & 68.5 & 141.6 & 0.0 & 47.0 & 100.4 & 0.0 & 8.842 & 0.000 \\
\hline Low alcohol content bevs (mL/day) & 1.1 & 4.1 & 0.0 & 1.4 & 10.5 & 0.0 & 1.6 & 7.3 & 0.0 & 1.518 & 0.220 \\
\hline High alcohol content bevs (mL/day) & 83.3 & 131.2 & 30.0 & 67.2 & 140.5 & 0.0 & 45.5 & 97.8 & 0.0 & 8.516 & 0.000 \\
\hline
\end{tabular}

General linear models were used to estimate multivariate means for food consumption across lifestyle patterns adjusted for age and energy intake. 
Table 4. Prevalence odds ratios (POR) of obesity in men and women, according to age group, level of education, geographical area, and lifestyle pattern.

\begin{tabular}{|c|c|c|c|c|c|c|c|c|}
\hline & \multicolumn{4}{|c|}{ Men } & \multicolumn{4}{|c|}{ Women } \\
\hline & \multirow{2}{*}{ POR } & \multicolumn{2}{|c|}{ 95\% C.I.POR } & \multirow{2}{*}{$p$} & \multirow{2}{*}{ POR } & \multicolumn{2}{|c|}{ 95\% C.I.POR } & \multirow{2}{*}{$p$} \\
\hline & & Lower & Upper & & & Lower & Upper & \\
\hline \multicolumn{9}{|l|}{ Age group } \\
\hline 50-64 years & & & & 0.000 & & & & 0.000 \\
\hline $18-30$ years & 0.29 & 0.17 & 0.48 & 0.000 & 0.29 & 0.16 & 0.52 & 0.000 \\
\hline $31-49$ years & 0.61 & 0.42 & 0.89 & 0.011 & 0.52 & 0.35 & 0.78 & 0.002 \\
\hline \multicolumn{9}{|l|}{ Level of education } \\
\hline High & & & & 0.293 & & & & 0.000 \\
\hline Primary or less & 1.41 & 0.87 & 2.28 & 0.162 & 3.11 & 1.74 & 5.58 & 0.000 \\
\hline Secondary & 1.08 & 0.69 & 1.69 & 0.730 & 1.85 & 1.06 & 3.24 & 0.031 \\
\hline \multicolumn{9}{|l|}{ Geographical area } \\
\hline South & & & & 0.017 & & & & 0.704 \\
\hline North-northwest & 1.13 & 0.65 & 1.99 & 0.658 & 0.83 & 0.45 & 1.50 & 0.532 \\
\hline Eastern-Mediterranean & 1.99 & 1.25 & 3.16 & 0.003 & 1.11 & 0.70 & 1.76 & 0.672 \\
\hline Center & 1.45 & 0.88 & 2.40 & 0.141 & 0.88 & 0.51 & 1.52 & 0.649 \\
\hline \multicolumn{9}{|l|}{ Lifestyle pattern } \\
\hline Poor diet-low physical activity-sedentary lifestyle pattern & & & & 0.058 & & & & 0.648 \\
\hline Mixed diet-physically active-low sedentary lifestyle pattern & 0.52 & 0.29 & 0.93 & 0.027 & 1.16 & 0.48 & 2.80 & 0.738 \\
\hline Not poor diet-low physical activity-low sedentary lifestyle pattern & 0.92 & 0.59 & 1.44 & 0.726 & 1.30 & 0.75 & 2.25 & 0.358 \\
\hline
\end{tabular}

Note: Binary logistic regression models adjusted for age group, educational level, geographical area, and energy intake. 


\section{Discussion}

In this cross-sectional study conducted in a random sample of Spanish adult population aged 18-64 years, four dietary patterns were characterized by factor analysis and subsequently used to identify three different lifestyle patterns based on the DPs, physical activities, sedentary time, and sleeping and smoking habits, using cluster analyses: a "Mixed diet-physically active-low sedentary lifestyle pattern", a "Not poor diet-low physical activity-low sedentary lifestyle pattern" which included 63.3\% of the sample, and a "Poor diet-low physical activity-sedentary lifestyle pattern". A higher proportion of people aged 18-30 years was classified into the "Poor diet-low physical activity-sedentary lifestyle pattern". The prevalence odds ratio for obesity in men allocated in the "Mixed diet-physically active-low sedentary lifestyle pattern" was significantly lower compared to those in the "Poor diet-low physical activity-sedentary lifestyle pattern".

Analysis of dietary patterns has become an important approach in food consumption studies and nutritional epidemiology $[11,27,28]$ Researchers report different methodological approaches and procedures to identify diet and lifestyle patterns, such as a priori defined patterns or data-driven, identified using principal components (PCA) [29], factor analysis [11,30], cluster analysis [31-33] and, more recently, hybrid methods [2]. Some pieces of research have identified lifestyle patterns associated to specific phenotypes and obesity in several population groups [34,35] and contributed to unravel the consequences that different diets may have on health [2,11,35-38].

In this study factor analysis was used to identify dietary patterns that were later used in cluster analysis. In the ANIBES study four DPs were singled out in adults. Similar DPs have been reported [37]. In Spain, in the context of the DORICA study, a pooled analysis of population-based cross-sectional surveys conducted between 1990 and 2000, using cluster analysis of three different DPs were characterized. A "Meat rich DP" with higher consumption of meat, cereals, and potatoes, an "Unbalanced DP" with higher consumption of milk and low consumption of vegetables and cereals, and a "Mediterranean DP" with higher consumption of vegetables, fruit, fish, and olive oil. The "Unbalanced $D P$ " was associated with higher alcohol consumption, inadequate fruit and vegetable consumption and poor physical activity. This pattern was associated to higher prevalence odds ratio for metabolic syndrome. Conversely, the "Mediterranean $D P$ " was associated with better fat intake quality profile and fiber intake [19].

More recently other authors have identified two DPs using factor analysis in Spanish adults in the context of Food4Me Project. A pattern with higher consumption of fast and processed foods, potatoes, red and white meats, processed cereals, and low consumption of fruit and vegetables, directly associated with BMI. A second DP showed higher consumption of eggs, fish, legumes, nuts, low-calorie beverages, oil, vegetables, and white meat [8].

Despite cross-sectional studies not allowing for causal inference, food patterns with higher energy density and low fiber content have been associated with higher obesity prevalence rates [2]. In longitudinal studies, such as the Baltimore Study, dietary patterns high in reduced-fat dairy, high fiber grains and cereals, vegetables and fruits, and low in meats, sugar-sweetened soft drinks, refined grains, and high fat dairy products, were significantly associated with lower weight gain, prospectively [39]. An energy-dense, high-saturated fat and low-fiber dietary pattern with high loadings of fast foods and snacks and low loadings of fruits and vegetables was shown to increase body weight, waist circumference, blood pressure, serum insulin, and lipid profile during a 10-year follow-up in severely obese Swedish adults [40]. In line with this, in adult Canadians a strong and consistent relation between an energy-dense, high-fat, low-fiber density dietary pattern and the risk of obesity was observed, significant in different population subgroups [2].

In the ANIBES study, $45.6 \%$ of male and $27.5 \%$ of female Spanish adults did not meet the recommendation of $150 \mathrm{~min}$ /week of moderate PA. In addition, $56.2 \%$ of male and $74 \%$ of female adults did not meet the recommendations for vigorous PA [13]. In this study we analyzed if these physical activity behaviors combined in a meaningful way with other energy balance-related behaviors, DPs, and lifestyles. We also used this procedure to identify dietary and lifestyle patterns in Spanish 
children and adolescents and identified two patterns: an "Unhealthier lifestyle pattern" a combination of low physical activity and poorer diet, and a "Healthier lifestyle pattern" which combined high physical activity, low sedentary behavior, longer sleep duration, and healthier diet.

Studies analyzing individual behaviors have found healthier eating habits, such as adequate fruit and vegetable consumption, to be more prevalent among women than in men [41], but adherence to physical activity recommendations were more prevalent in men than in women [42]. In line with the above, in this study "Snack DP" mean scores adjusted for age and energy intake were significantly

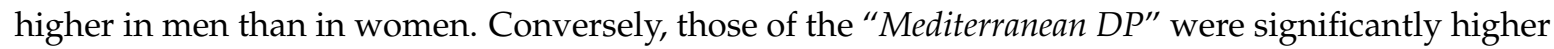
in women. Men and women classified in the "Mixed diet-physically active-low sedentary lifestyle pattern" showed the highest mean scores for the "Mediterranean DP". However, men in that lifestyle pattern showed the highest mean scores for the "Snack DP", as well. The highest mean scores for the "Dairy sweet $D P^{\prime \prime}$ were observed in men and women classified into the "Poor diet-low physical activity-sedentary lifestyle pattern".

In this study a significantly higher proportion of men was classified into the "Mixed diet-physically active-low sedentary lifestyle pattern" (71.9\%), while significantly more women were classified into the "Not poor diet-low physical activity-low sedentary lifestyle pattern" (58.5\%). Men classified in the "Mixed diet-physically active-low sedentary lifestyle pattern" were less likely to be obese (POR 0.52; 95\% IC 0.29-0.93), compared to those in the "Poor diet-low physical activity-sedentary lifestyle pattern". Cassidy et al. reported obese adults to be two to five times more likely to report an "unhealthy phenotype", a combination of low physical activity, high TV viewing, and poor sleep duration compared to normal weight adults in the UK Biobank [43].

A significantly higher proportion of women classified into the "Mixed diet-physically active-low sedentary lifestyle pattern" had a high educational level (35.6\%). Women with a low educational level were more likely to be obese compared to those highly educated. Clustering and co-occurrence of multiple risk behaviors has been reported to be more likely in men and women with lower education or intermediate educational level compared with those with higher education [44].

As shown in this study, individuals may follow a variety of unhealthy lifestyle behaviors which combine to favor weight gain, or mixtures of healthy and unhealthy practices. The findings in this study underline the importance of designing and implementing interventions that address multiple health risk habits, considering lifestyle patterns, clustering of risk behaviors, and associated determinants. It is recognized that interventions which target more than one risk behavior have the potential for greater health benefits, enhance health promotion opportunities and can contribute to reduce health care costs. Such an approach allows for more adequate tailoring of interventions to participants' profiles and helps to address health inequalities, reinforcing policies to ensure interventions accessible to socially-disadvantaged groups.

The ANIBES study has several strengths which include the careful design, protocol, and methodology used for dietary data collection and validated questionnaires used to collect information on physical activity which have shown good reliability and reproducibility. The study was conducted on a random population sample of the Spanish population aged 9-75 years. A limitation of this study is its cross-sectional design, which provides evidence for associations but not causal relationships. Measures of physical activity relied on self-reports and could be biased, although a careful multistep quality control procedure was implemented to minimize bias. In addition, we used factor analysis to identify DPs based on food consumption information collected by three-day food records and one 24-h recall, considering food groups to further collapse dietary intake data and avoid missing data from non-consumers of episodically consumed foods. Finally, both factor analysis and cluster analysis are procedures commonly used to identify DPs and analyze clustering of lifestyles. However, those procedures rely on several subjective decisions that may influence outcomes regarding the number and type of patterns and clusters identified. 


\section{Conclusions}

Four dietary patterns were characterized in Spanish adults aged 18-64 years, one of them closer to the Mediterranean DP. These patterns were subsequently used to identify three different lifestyle patterns: a "Mixed diet-physically active-low sedentary lifestyle pattern", a "Not poor diet-low physical activity-low sedentary lifestyle pattern" which included 63.3\% of the sample, and a "Poor diet-low physical activity-sedentary lifestyle pattern". A higher proportion of people aged 18-30 years was classified into the "Poor diet-low physical activity-sedentary lifestyle pattern". The prevalence odds ratio for obesity in men allocated in the "Mixed diet-physically active-low sedentary lifestyle pattern" was significantly lower compared to those in the "Poor diet-low physical activity-sedentary lifestyle pattern". Although prospective research on large population samples would be desirable to further analyze and track over time the lifestyle patterns and how they influence the development of overweight and obesity, these behavior patterns are helpful to identify specific issues in population subgroups and inform intervention strategies. Further research is needed on factors associated with lifestyle patterns to gain insight in the population subgroups at higher risk. The findings in this study underline the importance of designing and implementing interventions that address multiple health risk practices, considering lifestyle patterns and associated determinants.

Supplementary Materials: The following are available online at www.mdpi.com/2072-6643/9/6/606/s1, Table S1: Food groups and subgroups in the ANIBES Study, Table S2: Characteristics of the sample analyzed, Figure S1: Final cluster center scores for lifestyle patterns identified in Spanish adults aged 18-64 years in the ANIBES study.

Acknowledgments: The authors would like to thank Coca-Cola Iberia and IPSOS for its support and technical advice, particularly Rafael Urrialde and Javier Ruiz.

Author Contributions: C.P.R. analyzed the data and drafted the manuscript. M.G. contributed to draft the manuscript. J.A. contributed to the analysis and wrote the manuscript. This author is a member of the Scientific Advisory Board of the ANIBES study and, together with the other members, was responsible for careful review of the protocol, design, and methodology. This author provided continuous scientific advice for the study and for the interpretation of results. A.G., M.G.G., R.M.O. and L.S.M. are members of the Scientific Advisory Board of the ANIBES study, and were responsible for careful review of the protocol, design, and methodology. These authors provided continuous scientific advice for the study and for the interpretation of results. These authors also critically reviewed the manuscript. G.V.M., Principal Investigator of the ANIBES study, was responsible for the design, protocol, methodology, and follow-up checks of the study. All authors approved the final version of the manuscript.

Conflicts of Interest: The ANIBES study was financially supported by a grant from Coca-Cola Iberia through an agreement with the Spanish Nutrition Foundation (FEN). The funding sponsors had no role in the design of the study, in the collection, analyses, or interpretation of the data; in the writing of the manuscript, and in the decision to publish the results. The authors declare no conflict of interest.

\section{References}

1. Margetts, B. Feedback on WHO/FAO global report on diet, nutrition and prevention of chronic diseases (NCD). Public Health Nutr. 2003, 6, 423-424. [CrossRef] [PubMed]

2. Jessri, M.; Wolfinger, R.D.; Lou, W.Y.; L'Abbé, M.R. Identification of dietary patterns associated with obesity in a nationally representative survey of Canadian adults: Application of a priori, hybrid, and simplified dietary pattern techniques. Am. J. Clin. Nutr. 2017, 105, 669-684. [CrossRef] [PubMed]

3. López-Sobaler, A.M.; Aparicio, A.; Aranceta-Bartrina, J.; Gil, A.; González-Gross, M.; Serra-Majem, L.; Varela-Moreiras, G.; Ortega, R.M. Overweight and General and Abdominal Obesity in a Representative Sample of Spanish Adults: Findings from the ANIBES Study. Biomed Res. Int. 2016, 2016, 8341487. [CrossRef] [PubMed]

4. Basterra-Gortari, F.J; Bes-Rastrollo, M.; Ruiz-Canela, M.; Gea, A.; Martinez-Gonzalez, M.A. Prevalencia de obesidad y diabetes en adultos españoles, 1987-2012. Med. Clin. 2017, 148, 250-256. [CrossRef] [PubMed]

5. Eguaras, S.; Toledo, E.; Hernández-Hernández, A.; Cervantes, S.; Martínez-González, M.A. Better Adherence to the Mediterranean Diet Could Mitigate the Adverse Consequences of Obesity on Cardiovascular Disease: The SUN Prospective Cohort. Nutrients 2015, 7, 9154-9162. [CrossRef] [PubMed] 
6. Lacoppidan, S.A.; Kyrø, C.; Loft, S.; Helnæs, A.; Christensen, J.; Hansen, C.P.; Dahm, C.C.; Overvad, K.; Tjønneland, A.; Olsen, A. Adherence to a Healthy Nordic Food Index Is Associated with a Lower Risk of Type-2 Diabetes-The Danish Diet, Cancer and Health Cohort Study. Nutrients 2015, 7, 8633-8644. [CrossRef] [PubMed]

7. GBD 2015 Risk Factors Collaborators. Global, regional, and national comparative risk assessment of 79 behavioral, environmental and occupational, and metabolic risks or clusters of risks, 1990-2015: A systematic analysis for the Global Burden of Disease Study 2015. Lancet 2016, 388, 1659-1724.

8. San-Cristobal, R.; Navas-Carretero, S.; Celis-Morales, C.; Brennan, L.; Walsh, M.; Lovegrove, J.A.; Daniel, H.; Saris, W.H.M.; Traczyk, I.; Manios, Y.; et al. Analysis of Dietary Pattern Impact on Weight Status for Personalised Nutrition through On-Line Advice: The Food4Me Spanish Cohort. Nutrients 2015, 7, 9523-9537. [CrossRef] [PubMed]

9. Brown, R.C.; Tey, S.L.; Gray, A.R.; Chisholm, A.; Smith, C.; Fleming, E.; Parnell, W. Association of Nut Consumption with Cardiometabolic Risk Factors in the 2008/2009 New Zealand Adult Nutrition Survey. Nutrients 2015, 7, 7523-7542. [CrossRef] [PubMed]

10. Kremers, S.P. Theory and practice in the study of influences on energy balance-related behaviors. Patient Educ. Couns. 2010, 79, 291-298. [CrossRef] [PubMed]

11. Mikkilä, V.; Räsänen, L.; Raitakari, O.T.; Pietinen, P.; Viikari, J. Consistent dietary patterns identified from childhood to adulthood: The Cardiovascular Risk in Young Finns Study. Br. J. Nutr. 2005, 93, 923-931. [CrossRef] [PubMed]

12. Pronk, N.P.; Anderson, L.H.; Crain, A.L.; Martinson, B.C.; O'Connor, P.J.; Sherwood, N.E.; Whitebird, R.R. Meeting recommendations for multiple healthy lifestyle factors. Prevalence, clustering, and predictors among adolescent, adult, and senior health plan members. Am. J. Prev. Med. 2004, 27, 25-33. [CrossRef] [PubMed]

13. Mielgo-Ayuso, J.; Aparicio-Ugarriza, R.; Castillo, A.; Ruiz, E.; Ávila, J.M.; Aranceta-Batrina, J.; Gil, Á.; Ortega, R.M.; Serra-Majem, L.; Varela-Moreiras, G.; et al. Physical Activity Patterns of the Spanish Population Are Mostly Determined by Sex and Age: Findings in the ANIBES Study. PLoS ONE 2016, 11, e0149969. [CrossRef] [PubMed]

14. Hunsberger, M.; Mehlig, K.; Börnhorst, C.; Hebestreit, A.; Moreno, L.; Veidebaum, T.; Kourides, Y.; Siani, A.; Molnar, D.; Sioen, I.; et al. Dietary Carbohydrate and Nocturnal Sleep Duration in Relation to Children's BMI: Findings from the IDEFICS Study in Eight European Countries. Nutrients 2015, 7, 10223-10236. [CrossRef] [PubMed]

15. Cao, Y.; Wittert, G.; Taylor, A.W.; Adams, R.; Shi, Z. Associations between Macronutrient Intake and Obstructive Sleep Apnoea as Well as Self-Reported Sleep Symptoms: Results from a Cohort of Community Dwelling Australian Men. Nutrients 2016, 8, 207. [CrossRef] [PubMed]

16. El Ansari, W.; Suominen, S.; Berg-Beckhoff, G. Is Healthier Nutrition Behaviour Associated with Better Self-Reported Health and Less Health Complaints? Evidence from Turku, Finland. Nutrients 2015, 7, 8478-8490. [CrossRef] [PubMed]

17. Ruiz, E.; Ávila, J.M.; Castillo, A.; Valero, T.; del Pozo, S.; Rodriguez, P.; Aranceta Bartrina, J.; Gil, A.; González-Gross, M.; Ortega, R.M.; et al. The ANIBES Study on Energy Balance in Spain: Design, protocol and methodology. Nutrients 2015, 7, 970-998. [CrossRef] [PubMed]

18. Ruiz, E.; Ávila, J.M.; Valero, T.; del Pozo, S.; Rodriguez, P.; Aranceta-Bartrina, J.; Gil, A.; González-Gross, M.; Ortega, R.M.; Serra-Majem, L.; et al. Energy Intake, Profile, and Dietary Sources in the Spanish Population: Findings of the ANIBES Study. Nutrients 2015, 7, 4739-4762. [CrossRef] [PubMed]

19. Aranceta Bartrina, J.; Pérez-Rodrigo, C.; Serra-Majem, L.; Tur Marí, J.A.; Mataix Verdú, J.; Tojo Sierra, R.; Ribas Barba, L.; Martinez de Vitoria, E.; Millán Núñez-Cortés, J. Dieta y riesgo cardiovascular en España: Estudio DORICA (III). In Dieta y Riesgo Cardiovascular. Estudio DORICA II; Aranceta, J., Foz, M., Gil, B., Jover, E., Mantilla, T., Millán, J., Monereo, S., Moreno, B., Eds.; Médica Panamericana: Madrid, Spain, 2007; pp. 121-162.

20. Moreiras, O.; Carbajal, A.; Cabrera, L.; Cuadrado, C. Tablas de Composición de Alimentos, 15th ed.; Pirámide: Madrid, Spain, 2011.

21. Roman-Viñas, B.; Serra-Majem, L.; Hagströmer, M.; Ribas-Barba, L.; Sjöström, M.; Segura-Cardona, R. International physical activity questionnaire: Reliability and validity in a Spanish population. Eur. J. Sport Sci. 2010, 10, 297-304. [CrossRef] 
22. IPAQ. Guidelines for Data Processing and Analysis of the International Physical Activity Questionnaire (IPAQ)—Short and Long Forms. November 2005. Available online: https://sites.google.com/site/theipaq/ scoring-protocol (accessed on 27 October 2015).

23. Bauman, A.; Ainsworth, B.E.; Bull, F.; Craig, C.L.; Hagströmer, M.; Sallis, J.F.; Pratt, M.; Sjöström, M. Progress and Pitfalls in the Use of the International Physical Activity Questionnaire (IPAQ) for Adult Physical Activity Surveillance. J. Phys. Act. Health 2009, 6, S5-S8. [CrossRef] [PubMed]

24. Marfell-Jones, M.; Olds, T.; Stewart, A.; Carter, L. International Standards for Anthropometric Assessment; International Society for the Advancement of Kinanthropometry: Potchefstroom, South Africa, 2006; pp. 1-137.

25. Newby, P.K.; Tucker, K.L. Empirically derived eating patterns using factor or cluster analysis: A review. Nutr. Rev. 2004, 62, 177-203. [CrossRef] [PubMed]

26. Everitt, B.S.; Landau, S.; Leese, M.; Stahl, D. Cluster Analysis, 5th ed.; JohnWiley \& Sons, Ltd.: West Sussex, UK, 2011.

27. Hu, F.B. Dietary pattern analysis: A new direction in nutritional epidemiology. Curr. Opin. Lipidol. 2002, 13, 3-9. [CrossRef] [PubMed]

28. Hodge, A.; Bassett, J. What can we learn from dietary pattern analysis? Public Health Nutr. 2016, 19, $191-194$. [CrossRef] [PubMed]

29. Varraso, R.; Garcia-Aymerich, J.; Monier, F.; Le Moual, N.; De Batlle, J.; Miranda, G.; Pison, C.; Romieu, I.; Kauffmann, F.; Maccario, J. Assessment of dietary patterns in nutritional epidemiology: Principal component analysis compared with confirmatory factor analysis. Am. J. Clin. Nutr. 2012, 96, 1079-1092. [CrossRef] [PubMed]

30. Wadolowska, L.; Kowalkowska, J.; Czarnocinska, J.; Jezewska-Zychowicz, M.; Babicz-Zielinska, E. Comparing dietary patterns derived by two methods and their associations with obesity in Polish girls aged 13-21 years: The cross-sectional GEBaHealth study. Perspect. Public Health 2017, 137, 182-189. [CrossRef] [PubMed]

31. Wirfalt, A.K.; Jeffery, R.W. Using cluster analysis to examine dietary patterns: Nutrient intakes, gender, and weight status differ across food pattern clusters. J. Am. Diet. Assoc. 1997, 97, 272-279. [CrossRef]

32. Leech, R.M.; McNaughton, S.A.; Timperio, A. The clustering of diet, physical activity and sedentary behavior in children and adolescents: A review. Int. J. Behav. Nutr. Phys. Act. 2014, 11, 4. [CrossRef] [PubMed]

33. Pérez-Rodrigo, C.; Gil, A.; González-Gross, M.; Ortega, R.M.; Serra-Majem, L.; Varela-Moreiras, G.; Aranceta-Bartrina, J. Clustering of Dietary Patterns, Lifestyles, and Overweight among Spanish Children and Adolescents in the ANIBES Study. Nutrients 2016, 8, 11. [CrossRef] [PubMed]

34. Allès, B.; Samieri, C.; Lorrain, S.; Jutand, M.A.; Carmichael, P.H.; Shatenstein, B.; Gaudreau, P.; Payette, H.; Laurin, D.; Barberger-Gateau, P. Nutrient Patterns and Their Food Sources in Older Persons from France and Quebec: Dietary and Lifestyle Characteristics. Nutrients 2016, 8, 225. [CrossRef] [PubMed]

35. Blondin, S.A.; Mueller, M.P.; Bakun, P.J.; Choumenkovitch, S.F.; Tucker, K.L.; Economos, C.D. Cross-Sectional Associations between Empirically-Derived Dietary Patterns and Indicators of Disease Risk among University Students. Nutrients 2016, 8, 3. [CrossRef] [PubMed]

36. Appannah, G.; Pot, G.K.; Huang, R.C.; Oddy, W.H.; Beilin, L.J.; Mori, T.A.; Jebb, S.A.; Ambrosini, G.L. Identification of a dietary pattern associated with greater cardiometabolic risk in adolescence. Nutr. Metab. Cardiovasc. Dis. 2015, 25, 643-650. [CrossRef] [PubMed]

37. Bell, L.K.; Edwards, S.; Grieger, J.A. The Relationship between Dietary Patterns and Metabolic Health in a Representative Sample of Adult Australians. Nutrients 2015, 7, 6491-6505. [CrossRef] [PubMed]

38. Shu, L.; Zheng, P.F.; Zhang, X.Y.; Si, C.J.; Yu, X.L.; Gao, W.; Zhang, L.; Liao, D. Association between Dietary Patterns and the Indicators of Obesity among Chinese: A Cross-Sectional Study. Nutrients 2015, 7, 7995-8009. [CrossRef] [PubMed]

39. Newby, P.K.; Muller, D.; Hallfrisch, J.; Andres, R.; Tucker, K.L. Food patterns measured by factor analysis and anthropometric changes in adults. Am. J. Clin. Nutr. 2004, 80, 504-513. [PubMed]

40. Johns, D.J.; Lindroos, A.K.; Jebb, S.A.; Sjostrom, L.; Carlsson, L.M.; Ambrosini, G.L. Dietary patterns, cardiometabolic risk factors, and the incidence of cardiovascular disease in severe obesity. Obesity 2015, 23, 1063-1070. [CrossRef] [PubMed] 
41. Mackenbach, J.D.; Brage, S.; Forouhi, N.G.; Griffin, S.J.; Wareham, N.J.; Monsivais, P. Does the importance of dietary costs for fruit and vegetable intake vary by socioeconomic position? Br. J. Nutr. 2015, 114, 1464-1470. [CrossRef] [PubMed]

42. de Azevedo Barros, M.B.; Guimarães Lima, M.; Barbosa Medina, L.P.; Landman Szwarcwald, C.; Carvalho Malta, D. Social inequalities in health behaviors among Brazilian adults: National Health Survey, 2013. Int. J. Equity Health 2016, 15, 148. [CrossRef] [PubMed]

43. Cassidy, S.; Chau, J.Y.; Catt, M.; Bauman, A.; Trenell, M.I. Low physical activity, high television viewing and poor sleep duration cluster in overweight and obese adults; a cross-sectional study of 398,984 participants from the UK Biobank. Int. J. Behav. Nutr. Phys. Act. 2017, 14, 57. [CrossRef] [PubMed]

44. Meader, N.; King, K.; Moe-Byrne, T.; Wright, K.; Graham, H.; Petticrew, M.; Power, C.; White, M.; Sowden, A.J. A systematic review on the clustering and co-occurrence of multiple risk behaviours. BMC Public Health 2016, 16, 657. [CrossRef] [PubMed]

(C) 2017 by the authors. Licensee MDPI, Basel, Switzerland. This article is an open access article distributed under the terms and conditions of the Creative Commons Attribution (CC BY) license (http:/ / creativecommons.org/licenses/by/4.0/). 